\title{
La formación universitaria en psicología en Argentina: perspectivas actuales y desafíos a la luz de la historia*
}

University Education in Psychology in Argentina: Current Perspectives and Challenges from History

\author{
HugO KLAPPENBACH ** \\ Universidad Nacional de San Luis \\ Consejo Nacional de Investigaciones Científicas \\ y Técnicas, Argentina
}

doi:10.11144/Javeriana.upsy14-3.fupa

Para citar este artículo: Klappenbach, H. (2015). La formación universitaria en psicología en Argentina: perspectivas actuales y desafíos a la luz de la historia. Universitas Psychologica, 14(3), 937-960. http:// dx.doi.org/10.11144/Javeriana.upsy14-3.fupa

* Artículo de investigación. Agradezco a mi equipo de investigación en el cual hemos discutido en distintas oportunidades estas problemas y a los evaluadores anónimos por sus críticas sugerentes y por su lectura atenta al manuscrito original.

** Investigador Principal del Consejo Nacional de Investigaciones Científicas y Técnicas (CONICET) y Profesor Titular Efectivo en Historia de la Psicología en la Universidad Nacional de San Luis (UNSL).E-mail: hklappen@unsl.edu.ar
RESUMEN

El presente trabajo analiza los orígenes de la enseñanza de la psicología en Argentina, para centrarse luego en la organización de las carreras o programas de psicología en el país. La finalidad es realizar un inventario completo de los centros universitarios que incluyen carreras de psicología en su oferta educativa. Posteriormente, se analiza el impacto del proceso de acreditación obligatoria de carreras de psicología de grado, iniciado en 2009 y parcialmente finalizado en diciembre de 2013. También se catalogan las carreras de posgrado y las organizaciones de la psicología, las cuales revelan el sesgo profesionalista y clinicista que ha caracterizado a la psicología argentina desde comienzos de la década de 1960.

Palabras clave

formación en Psicología; carreras de grado; carreras de posgrado; instituciones profesionales; instituciones científicas; Argentina

\section{A B S T R A C T}

This paper analyzes the beginnings of Psychology Education in Argentina and then focusing on the organization of undergraduate programs in the whole country. The aim is to carry out a complete updating information of all universities including psychology programs. Subsequently, the impact of the process of accreditation of undergraduate psychology programs is analyzed. Accreditation began in 2009 and it is not finish at all. On the other hand, graduate programs and organizations of psychology are also examined. It also confirms the professional and clinic bias that characterized Argentina psychology since the early 1960s.

Keywords

Psychology education; undergraduate programs; graduate programs; scientific Psychology organizations; professional Psychology organizations; Argentina 


\section{Introducción}

La enseñanza de la psicología en Argentina se remonta a finales del siglo XIX. Al comienzo del siglo $\mathrm{XX}$, existían cursos de psicología destinados a la formación de maestros normalistas y profesores en la Universidad Nacional de La Plata y la Universidad de Buenos Aires, a la formación de médicos en la Universidad Nacional de Córdoba y a la formación de abogados en la Universidad de Santa Fe y en Buenos Aires (Ferrari, 2013; Ingenieros, 1910; Klappenbach, 2006).

Es decir, la enseñanza temprana de la psicología en Argentina no estaba destinada a la formación de futuros graduados en psicología. Más todavía, las primeras carreras o programas en el campo epistémico de la psicología es un fenómeno posterior y en un primer momento se orientaron a la psicotecnia y la orientación profesional. En 1928, se había organizado la Escuela de Consejeros de Orientación Profesional en el Instituto de Psicotecnia y Orientación Profesional, bajo la dirección de Carl Jessinghaus en la que llegarían a graduarse 56 consejeros antes de su cierre luego del golpe militar de 1930 (Edelmuth, 1997).

En 1950, en la Universidad Nacional de Tucumán, Benjamin Aybar organizó la Licenciatura en Psicotecnia y Orientación Profesional. Por su parte, en la Universidad Nacional del Litoral, en la ciudad de Rosario se organizó la Carrera de Asistente en Psicotécnica en 1953 (Klappenbach, 2006).

El primer programa universitario que explícitamente incorporó el nombre psicología se organizó en 1953, en la Universidad Nacional de Cuyo, en la ciudad de San Luis. Allí, Plácido Horas organizó la Especialización en Psicología. Sin embargo, el objetivo de la misma era formar los recursos humanos necesarios para trabajar en la Dirección de Psicología Educacional y Orientación Profesional. Es decir, la articulación entre psicología y psicotecnia y orientación profesional fue constante desde finales de 1920 hasta mediados de la década de 1950.

En 1954, en San Miguel de Tucumán, se reunió el Primer Congreso Argentino de Psicología. La comisión Perspectivas y necesidades de los estudios psicológicos en nuestro país, cuyos relatores fueron Plácido Horas y Eugenio Pucciarelli, recomendó:

El Primer Congreso Argentino de Psicología declara la necesidad de crear la carrera universitaria del psicólogo profesional con arreglo a las siguientes condiciones: I. Se establecerá como sección autónoma en las Facultades de carácter humanístico, aprovechando los institutos ya existentes y la enseñanza que se imparte en esas y en otras Facultades que puedan ofrecer su colaboración (Medicina, Derecho, Ciencias Económicas, etc.); II. La carrera comprenderá un plan completo de asignaturas teóricas y la debida intensificación práctica en las distintas especialidades de la profesión psicológica, otorgando los títulos de Licenciado en Psicología (previa tesis de Licenciatura) y de Doctor en Psicología (previa tesis de Doctorado). (Anónimo, 1954, p. 122; la cursiva es nuestra)

Puede advertirse el sesgo claramente profesional que se pretendía de la nueva carrera universitaria. No se trataba de una carrera de psicología, sino de una carrera de psicólogo profesional. Como consecuencia de aquella recomendación en los diez años posteriores a aquel Congreso, entre 1954 y 1964, se organizaron las primeras 14 carreras de psicología en universidades argentinas: seis en universidades nacionales; seis en universidades privadas, las cuales comenzaron a ser autorizadas en el país luego de la caída del peronismo en 1955 y dos en instituciones educativas provinciales (véase Tabla 1).

Hay otras dos características que merecen señalarse de aquellos primeros programas de psicología. La primera, que las carreras fueron recomendadas dentro del contexto político-social dominado por el peronismo. Entre 1943 y 1954, Argentina había duplicado largamente la producción industrial, pasando de 59.765 establecimientos industriales a 148.371 (Lewis, 1990). En ese marco, la orientación profesional alcanzó rango constitucional en la reforma de 1949, cuyo artículo 37 (Cap. III - IV - numeral 3) establecía que:

La orientación profesional de los jóvenes, concebida como un complemento de la acción de instruir y 
TABLA 1

Carreras de Psicología en los primeros diez años (1955-1964)

\begin{tabular}{lll}
\hline Fecha de Creación & \multicolumn{1}{c}{ Ciudad } & \multicolumn{1}{c}{ Universidad } \\
\hline 1955 (abril) & Rosario & Univ. Nacional del Litoral. Luego de la caída del peronismo se reorganizó en \\
1956 (marzo) & Buenos Aires & Univ. De El Salvador (jesuita originariamente). \\
1957 (marzo) & Buenos Aires & Univ. de Buenos Aires \\
1958 (febrero) & San Luis & Univ. Nacional de Cuyo \\
1958 (noviembre) & La Plata & Univ. Nacional de La Plata \\
1958 (diciembre) & Córdoba & Univ. Nacional de Córdoba. En 1956 había comenzado una carrera de \\
1959 (agosto) & Tucumán & Univ. Nacional Tucumán \\
1959 (agosto) & Córdoba & Univ. Católica de Córdoba (jesuita) \\
& & Univ. Nacional de Mar del Plata. En 1960 comenzó como Programa del \\
1960 (mayo) & Mar del Plata & $\begin{array}{l}\text { Instituto de Ciencias de la Educación. In 1966 sería incorporado a la Univ. } \\
\text { Provincial de Mar del Plata. Fue cerrado en 1976 durante la dictadura militar y } \\
\text { reabierta en 1985 ya dentro de la Univ. Nacional. }\end{array}$ \\
1961 (marzo) & Buenos Aires & $\begin{array}{l}\text { Facultad Libre de Psicología. Anexada a la Univ. Católica Argentina hasta } \\
\text { septiembre de 1969. }\end{array}$ \\
1962 (marzo) & Buenos Aires & $\begin{array}{l}\text { Univ. del Museo Social Argentino } \\
1963 \text { (marzo) }\end{array}$ \\
Tucumán & Univ. del Norte Santo Tomás de Aquino \\
1963 (agosto) & Mendoza & $\begin{array}{l}\text { Facultad de Antropología Escolar. Administración General de Escuelas de la } \\
\text { provincia de Mendoza. Fue clausurada definitivamente en 1997. }\end{array}$ \\
1964 (marzo) & Buenos Aires & Univ. Argentina John F. Kennedy \\
\hline
\end{tabular}

Fuente: Klappenbach (2003), con modificaciones introducidas por Mercado (2006).

educar, es una función social que el Estado ampara y fomenta mediante instituciones que guíen a los jóvenes hacia las actividades para las que posean naturales aptitudes y capacidad, con el fin de que la adecuada elección profesional redunde en beneficio suyo y de la sociedad. (Constitución de la Nación Argentina, 1949, p. 23)

Por su parte, el Segundo Plan Quinquenal que pretendía orientar las políticas del Estado a partir del segundo gobierno de Perón en 1952, establecía el objetivo de establecer "correlaciones racionales entre la aptitud del trabajador y su ocupación, a fin de obtener los más altos índices de productividad y de retribución" (Ley 14.184 de 1952, p. 83; la cursiva es nuestra).

Sin embargo, con la excepción de la primera carrera de psicología en Rosario, las restantes carreras se organizaron luego del golpe militar que derrocó a Perón en 1955. Inclusive, la primera carrera no sobrevivió al clima político posterior a la caída del peronismo y fue reorganizada en 1956.
Y la segunda característica, es que aun cuando se organizaron todas aquellas carreras, no existía en el país ninguna reglamentación ni caracterización sobre el perfil que se pretendía de los nuevos profesionales. Eso generó, ya en los primeros años de instaladas las carreras de psicología y sobre todo a partir de 1960 cuando los primeros graduados comenzaron a egresar de las mismas, un intenso debate acerca del perfil del profesional o, como se lo conoció en aquellos años, un debate sobre el rol del psicólogo (Bricht et al., 1973; Klappenbach, 2006).

Es interesante destacar, en primer lugar, que para el funcionamiento de aquellas primeras carreras de psicología "ya había gente preparada, construida, con una dinámica propia" (Noé, 2005, p. 92). Y en segundo lugar, que no obstante su descalificación como carreras de una supuesta 'psicología académica' propiamente especulativa (Bleger, 1962), desde la década de 1940 por lo menos, paralelamente a la enseñanza en la universidad, se había desarrollado de manera sistemática toda una vertiente de psicología aplicada, principalmente en el campo escolar 
y laboral. Como ya hemos señalado, esa vertiente aplicada se desarrolló bajo la figura de psicotecnia y orientación profesional (Klappenbach, 2006). Es decir, la psicología aplicada anterior a la caída del peronismo no se había concentrado principalmente en el dominio de la salud mental ni en la práctica clínica, aun cuando hubiera existido un desarrollo en tales campos. En cambio, la psicología aplicada se había desarrollado fuerte y ampliamente en el dominio del trabajo y de la educación. Por otro lado, también puede constatarse que, desde el punto de vista teórico, la psicología aplicada previa a 1955 -es decir, la psicotecnia y la orientación profesional- no se apoyaba en el psicoanálisis.

En cualquier caso, lo que resulta de interés es que la psicología que se fue construyendo desde comienzos de la década de 1960 tanto en el espacio académico como en el campo aplicado, se caracterizó por una fuerte ruptura con todo el desarrollo de la psicotecnia y la orientación profesional. En esa dirección, se ha señalado que:

(...) aun cuando todavía no disponemos de investigaciones que hayan analizado suficientemente en qué medida la ruptura se sostuvo en la necesidad de superar por anacrónica aquella antigua psicología académica y en qué medida la ruptura se apoyó en un simple desconocimiento de muchas de las características de la misma, es posible que las dos cuestiones mencionadas, la vocación por el campo de la clínica y la salud mental y el interés en la apropiación del psicoanálisis, promovieron en los nuevos graduados en psicología una brusca ruptura con cualquier desarrollo anterior de la psicología. (Klappenbach \& Arrigoni, 2011, p. 50)

El desarrollo posterior de aquellas indefinidas carreras de psicología en las décadas siguientes ha sido bien analizado (Courel \& Talak, 2001; Dagfal, 2009; Klappenbach, 2000; Vezzetti, 2004; Vilanova, 1993). En general, se ha enfatizado el interés casi exclusivo en la práctica clínica y la hegomonía del psicoanálisis, aun cuando no se tratara de un psicoanálisis ortodoxo.

Con posterioridad a la recuperación democrática, desde 1984, bajo la presidencia de Raúl Alfonsín, ese perfil de formación fue legitimado a pedido de las instituciones profesionales de psicólogos y psicólogas. En agosto de 1985, la Secretaría de Asuntos Universitarios del Ministerio de Educación y Justicia dirigida por Hugo Storani, convocó a unas Jornadas de Trabajo para discutir los alcances de los títulos de Psicólogo y Licenciado en Psicología. En dicho encuentro tuvieron participación autoridades de las carreras de psicología tanto en universidades públicas como privadas junto con dirigentes de la Federación de Psicólogos de la República Argentina (FePRA), que nucleaba a las asociaciones y colegios de psicólogos de todo el país. En tal reunión se elaboró un proyecto de resolución sobre Incumbencias del título de psicología, que sería enseguida aprobado por medio de la Resolución ministerial 2447/85 que establecía los alcances del título de psicólogo para las áreas de la psicología clásicas (Klappenbach, 2000). En esa dirección, la aprobación del perfil del graduado de 1995 marca la culminación de un proceso de formación iniciado a finales de la década de 1950 o comienzos de 1960.

Por otro lado, el desarrollo posterior de las carreras o programas de psicología en Argentina, ha estado anudado al crecimiento y expansión del sistema universitario, especialmente en la primera mitad de la década de 1990:

Desde hace varias décadas el sistema de educación superior se encuentra en proceso de expansión, de diferenciación institucional y de distribución territorial. La demanda de escolarización superior ha sido creciente (...). (Pérez-Lindo, 2007, p. 19)

Gracias a las nuevas instituciones, y a la dinámica de expansión de las ya existentes con anterioridad a 1982, la oferta de programas duplica su número hasta superar las 2.000 carreras. Al mismo tiempo, el subsistema terciario no universitario también se expande hasta alcanzar una cifra aún no bien conocida de instituciones que se estima en unas 1.200. (Fanelli \& Balán, 1994, p. 18)

También se ha observado que a partir de 2007, en un contexto político diferente al de la década de 1990, también se inauguraron 12 nuevas uni- 
versidades, 9 de ellas nacionales y muchas de ellas en el llamado Gran Buenos Aires (Suasnábar \& Rovelli, 2011). Es posible que la expansión del sistema de educación superior continúe en el futuro, tanto debido a razones nacionales como regionales; a nivel nacional, debido a la promoción de la obligatoriedad de la educación secundaria y la ampliación del programa de becas tanto para el acceso y la permanencia de estudiantes de escasos recursos como para el estudio de carreras tecnológicas. A nivel regional, porque un fenómeno análogo se ha observado en Venezuela, Cuba, Brasil y Colombia (Perez-Rasetti \& Araujo, 2010). En cualquier caso, el fenómeno de expansión del sistema de educación superior ha sido observado para el caso particular de las carreras de psicología.

Estos procesos, propios de las instituciones latinoamericanas pero no ajenos a los de los países desarrollados, promovieron el aumento y la diversificación de la oferta frente al aumento matricular, en contextos de progresivo ajuste económico. (Di Doménico \& Piacente, 2003, p. 35)

En los últimos veinte años, el plantel de psicólogos en la región viene multiplicándose exponencialmente. En muchos países el número de universidades que imparten estudios de grado aumentó en gran medida. (Fernández-Álvarez, 2003, p. 13)

En efecto, una de las carreras universitarias que experimentó mayor crecimiento de programas fue la carrera de psicología. Mientras que en los casi 40 años que median entre 1954 y 1990 solamente se crearon carreras de psicología en 7 universidades públicas y 11 universidades privadas, en apenas 17 años, entre 1990 y 2007 se crearon carreras de psicología en 3 universidades públicas y 21 universidades privadas (Tablas 2, 3 y 4).

Un problema para el relevamiento de la totalidad de los programas de grado en psicología existentes en el país surge de la correcta determinación de cómo se caracterizan a carreras de una misma universidad, con un mismo plan de estudios, pero que se desarrollan en sedes, subsedes o extensiones áulicas diferentes.
Dos argumentos justifican que en este trabajo sean consideradas como carreras diferentes. El primer razonamiento es simplemente normativo o administrativo. En efecto, el artículo 8 de la resolución 343/09 del Ministerio de Educación, la cual estableció los estándares para la acreditación de carreras de grado de psicología en el país ha sido explícito al respecto:

Art. $8^{\circ}$ - Sin perjuicio del cumplimiento de otras normas legales o reglamentarias aplicables al caso, la oferta de cursos completos o parciales de alguna carrera correspondiente a los títulos mencionados en el artículo $1^{\mathrm{o}}$ que estuviere destinada a instrumentarse total o parcialmente fuera de la sede principal de la institución universitaria, será considerada como una nueva carrera. (Ministerio de Educación, 2009, p. 7; la cursiva es nuestra)

El artículo mencionado es coherente con la preocupación que ya en 1998 había manifestado el organismo responsable de la acreditación de grado y posgrado en el país, la Comisión Nacional de Evaluación y Acreditación Universitaria (CONEAU) en relación con la existencia de subsedes o extensiones áulicas. En dicho documento, la Comisión caracterizaba el fenómeno como anárquico y advertía que "debilita la calidad del sistema de educación superior por la insuficiencia en dichas subsedes del plantel académico, de la infraestructura física y bibliográfica y de otros servicios esenciales para el aprendizaje" (CONEAU, 1998, p. 1).

Otros estudios han coincidido en esa perspectiva cuestionadora, subrayando que no siempre la universidad que dicta una carrera total o parcialmente en una subsede hace público ese dato desde el punto de vista administrativo. Es decir, ha llegado a ocurrir que los estudiantes son inscriptos en los mismos registros estadísticos en los que se inscriben los estudiantes de la sede principal y el título puede llegar a omitir la mención a la extensión áulica (Pérez-Rasetti, 2008).

Con todo, el fenómeno admite interpretaciones diversas, toda vez que pueden señalarse numerosas modalidades y variantes de estas subsedes. Desde aquellas que implican asociaciones entre 
la universidad que dicta la carrera y un organismo público (por ejemplo una municipalidad), a otras que significan asociaciones con organizaciones del medio o con instituciones terciarias no universitarias, entre otras (Pérez Rasetti, 2008). En 2009 se advertía la existencia de 94 universidades en todo el país (46 públicas, 46 privadas, una provincial y una extranjera) y de 258 extensiones áulicas (148 nacionales y 110 privadas) sin ningún tipo de planificación (Zelaya, 2012).

En cualquier caso, la asociación entre instituciones universitarias y gobiernos municipales excede con creces al caso de la enseñanza de la psicología y es un fenómeno que se ha extendido a diversas carreras y que se desarrolla en universidades privadas y en universidades públicas por igual. Resulta de interés el análisis del caso de la Universidad Nacional de La Plata (UNLP), ya que corresponde a una universidad de larga trayectoria y que históricamente fue considerada entre las más prestigiosas del país por su hincapié temprano en la investigación y por el nivel de sus académicos (Biagini, 1992, Vol. 3). Entre 1990 y 2006, la UNLP llegó a implementar 30 sedes externas a la ciudad de La Plata en la que participaban 9 facultades (Marano, 2010). El caso de la implementación de carreras de psicología a través de sedes o subsedes o extensiones áulicas externas a la sede principal, también ha contribuido a expandir significativamente la oferta de carreras de psicología en nuestro país. Años atrás, la Universidad Nacional de Rosario dictó por extensión la carrera de psicología en Bariloche y en Junín, Provincia de Buenos Aires. La experiencia de dictados de carreras de psicología en Junín es interesante porque se hizo en el Centro Universitario de Junín que en 2002 condujo a la creación de la Universidad Nacional del Noroeste de la Provincia de Buenos Aires (UNNOBA) con sedes en Pergamino y Junín (Pérez-Rasetti, 2008).

El segundo razonamiento que justifica considerar que el mismo programa de estudio de una misma universidad dictado en sedes diferentes sean considerados como carreras diferentes se fundamenta en consideraciones teóricas, específicamente en la conceptualización de César Coll sobre los tres niveles de concreción del currículo (Coll, 1994). El primer nivel de concreción constituye el currículo prescripto (Calabresi \& Polanco, 2011). En esa dirección, está relacionado con aquellos aspectos que el diseño curricular establece explícitamente como perfil, objetivos y contenidos de las carreras. Desde el punto de vista de la teoría didáctica y de la teoría del currículo constructivista, es necesario advertir que la noción de contenidos no alude únicamente a los hechos y conceptos que deben ser aprendidos, sino también a otros dos elementos centrales: por una parte, los procedimientos, por la otra, las actitudes, valores y normas (Calabresi \& Polanco, 2011).

El segundo nivel de concreción consiste en "la contextualización y pormenorización” del currículo base prescripto (Coll, 1994, p. 134). Está relacionado, entonces, con las condiciones objetivas que determinan el contexto institucional del desarrollo de los planes de estudio, desde la infraestructura de recursos hasta la elaboración o incidencia de los factores de gestión, coordinación y evaluación curricular.

Por su parte, el tercer nivel de concreción del currículo se relaciona con la programación e implementación del plan de estudios en las prácticas docentes cotidianas y en tal sentido "son competencia y responsabilidad de cada profesor y constituyen el referente inmediato del currículum en acción, del currículum real que van a experimentar los alumnos y alumnas" (Coll, 1994, p. 134). Habitualmente, constituye el currículo en el aula, y más ampliamente en actividades prácticas, pasantías, Practicum e internados, etc.

Desde esa concepción, aun cuando en el primer nivel de concreción del currículo relacionado con el plan de estudios prescripto, pudiera llegar a ser idéntico en distintas sedes de una misma universidad, el segundo y el tercer nivel de concreción necesariamente se modifican en cada sede o subsede, aunque los docentes pudieran a llegar a ser los mismos.

Aclaradas estas dificultades, un relevamiento actualizado al 15 de agosto de 2014, revela la existencia de 41 universidades que ofrecen carrera de grado de psicología, 23 de las cuales son posteriores a 1990, es decir, más de la mitad de las carreras (56.09\%). 
TABLA 2

Universidades públicas que ofrecen carreras en psicología en Argentina

\begin{tabular}{lclc}
\hline Universidad Nacional & Inicio & Unidad Académica, sedes y lugar de emplazamiento & Título que otorga \\
\hline Universidad Nacional de Rosario & 1955 & Facultad de Psicología. 2 sedes: Rosario y Marcos Juarez * & Psicólogo \\
Universidad de Buenos Aires & 1957 & Facultad de Psicología. & Lic. en Psicología \\
Universidad Nacional de San Luis & 1958 & Facultad de Ciencias Humanas & Lic. en Psicología \\
Universidad Nacional de La Plata & 1958 & Facultad de Psicología. 2 sedes: La Plata y Chivilcoy *** & Lic. en Psicología \\
Universidad Nacional de Córdoba & 1958 & Facultad de Psicología & Lic. en Psicología \\
Universidad Nacional de Tucumán & 1959 & Facultad de Psicología & Psicólogo \\
Universidad Nacional de Mar del Plata & 1960 & Facultad de Psicología *** & Lic. en Psicología \\
Universidad Nacional de La Rioja & 2000 & Departamento de Humanidades & Lic. en Psicología \\
Universidad Nacional del Comahue & 2005 & Facultad de Ciencias de la Educación. Cipoletti & Organizacional \\
Universidad Autónoma de Entre Ríos (provincial) & 2006 & Facultad de Humanidades, Artes y Ciencias Sociales & Psicólogo \\
\hline
\end{tabular}

* La sede de Marcos Juárez ya no abre el ingreso para nuevos cursantes desde el año pasado y continuará hasta finalizar esa cohorte. También existió una extensión en Venado Tuerto, que ya no se dicta (C. Bonantini, comunicación personal, 11 de agosto de 2014).

** También existió una extensión áulica en Bolívar pero allí ya no volverá a abrirse el cursado de la carrera (Viguera, Ariel, comunicación personal, 31 de agosto de 2014)

*** También existió una en la sede en Tres Arroyos que ya no volverá a abrirse el cursado de la carrera (J. Vivas, comunicación personal, 18 de agosto de 2014).

Fuente: relevamiento del autor con base en datos públicos de las propias universidades, comunicaciones personales y Ministerio de Educación, Secretaría de Políticas Universitarias, Dirección Nacional de Gestión Universitaria (2014). Base de títulos oficiales. Recuperado de http://titulosoficiales.siu.edu.ar/ GestiónUniversitaria (2014). Base de títulos oficiales. Recuperado de http:// titulosoficiales.siu.edu.ar/

Considerando el tipo de gestión (público o privado), se observa que ofrecen carrera de grado de psicología 10 universidades públicas: 9 universidades nacionales y 1 provincial (véase Tabla 2). Una sola de ellas, la Universidad Nacional de La Rioja, otorga el título de Licenciado en Psicología Organizacional, caso único en el país y que por considerar que no estaba incluido en los alcances del título generalista en psicología, no se presentó al proceso de acreditación iniciado en el año 2011.

Por su parte, seis de esas 10 universidades otorgan el título de Licenciado en Psicología y sólo tres de ellas, las de Rosario, Tucumán y Comahue, el título de Psicólogo. Más allá de que históricamente la diferente denominación se correspondía con diferencias académicas y profesionales, desde el punto de vista administrativo, para las normativas universitarias argentinas ambas titulaciones han sido consideradas equivalentes. Así, la antigua resolución de incumbencias del año 1985 se denominaba oficialmente "Incumbencias de los títulos de Psicólogo y de Licenciado en Psicolo- gía" (Ministerio de Educación y Justicia, 1985). Y la actual Resolución 343/09 del Ministerio de Educación, especificaba en su primer artículo: "Aprobar los contenidos curriculares básicos, la carga horaria mínima, los criterios de intensidad de la formación práctica y los estándares para la acreditación de las carreras correspondientes a los títulos de Psicólogo y Licenciado en Psicología" (Ministerio de Educación, 2009, p. 7, la cursiva es nuestra).

Puede apreciarse en la Tabla 2 que mientras siete universidades nacionales y la universidad provincial dictan la carrera en una sola sede, dos universidades nacionales (La Plata y Rosario) dictan la carrera en dos subsedes o extensiones áulicas. Es decir, existen 12 carreras de psicología de universidades públicas.

Por su parte, existen 31 universidades privadas que ofrecen el dictado de la carrera de psicología, 20 de ellas posteriores a 1990 (Tablas 3 y 4). Es decir que casi las dos terceras parte de las universidades que dictan la carrera de psicología en universidades de gestión privada, la han implementado con 
TABLA 3

Universidades privadas que ofrecen carreras de Psicología (anteriores a 1983)

\begin{tabular}{|c|c|c|c|}
\hline Universidad Privada & Inicio & Unidad Académica, sedes y lugar de emplazamiento & Título que otorga \\
\hline Universidad del Salvador & 1956 & $\begin{array}{l}3 \text { sedes. Facultad de Psicología y Psicopedagogía: } \\
\text { CABA *, Pilar y Bahía Blanca }\end{array}$ & Lic. en Psicología \\
\hline Universidad Católica de Córdoba & 1959 & Facultad de Filosofía y Humanidades. Córdoba ** & Lic. en Psicología \\
\hline Pontificia Universidad Católica Argentina & $\begin{array}{l}1960 \\
1970\end{array}$ & $\begin{array}{l}3 \text { sedes. 1) Facultad de Psicología y Psicopedagogía, } \\
\text { CABA. 2) Fac. Humanidades y Cs. de la Educación, } \\
\text { Mendoza. 3) Fac. Teresa de Ávila, Paraná, Prov. Entre } \\
\text { Ríos **** }\end{array}$ & Lic. en Psicología \\
\hline Universidad del Museo Social Argentino & 1962 & Facultad de Ciencias Humanas. CABA & Lic. en Psicología \\
\hline Universidad del Norte Santo Tomás de Aquino & 1963 & $\begin{array}{l}2 \text { sedes: Facultad de Ciencias de la Salud. San Miguel } \\
\text { de Tucumán y Centro Univ. Concepción, Prov. Tuc. }\end{array}$ & Lic. en Psicología \\
\hline Universidad Argentina John F. Kennedy & 1964 & $\begin{array}{l}\text { Departamento de Psicología- Escuela de Psicología. } \\
3 \text { sedes: Colegio Central. CABA; Colegio San Isidro } \\
\text { Labrador y Colegio Lanús }\end{array}$ & Lic. en Psicología \\
\hline Universidad Católica de Salta & 1967 & Facultad de Artes y Ciencias. Salta & Lic. en Psicología \\
\hline Universidad de Belgrano & 1967 & Facultad de Humanidades. CABA & Lic. en Psicología \\
\hline Universidad del Aconcagua & 1968 & Facultad de Psicología. Mendoza & Lic. en Psicología \\
\hline Universidad Católica de Cuyo & 1970 & Facultad de Filosofía y Humanidades. San Juan***** & Lic. en Psicología \\
\hline Universidad Católica de La Plata & 1982 & Facultad de Humanidades. 2 sedes: La Plata y Rosario & Lic. en Psicología \\
\hline
\end{tabular}

* CABA: Ciudad Autónoma de Buenos Aires

** Cerrada entre 1976 y 2007.

*** La carrera en la UCA se origina en 1970; entre 1960 y 1969 la Facultad Libre de Psicología estuvo anexada a la Universidad Católica (M. Caamaño, comunicación personal).

**** Entre 2005 y 2011 también se dictó la carrera en la sede Río Cuarto que ya no volverá a abrirse.

Fuente: relevamiento del autor con base en datos públicos de las propias universidades, comunicaciones personales y Ministerio de Educación, Secretaría de Políticas Universitarias, Dirección Nacional de Gestión Universitaria (2014). Base de títulos oficiales. Recuperado de http://titulosoficiales.siu.edu.ar/

posterioridad a las reformas educativas de la década de 1990 (exactamente el 64.51\%).

Sin embargo, ello tampoco quiera decir que existan solamente 31 carreras de psicología. Porque mientras 16 de esas universidades privadas dictan la carrera en una sola sede, seis la dictan en dos sedes o subsedes, otras seis en tres sedes o subsedes y dos universidades en cinco sedes o subsedes diferentes. Por su parte, una universidad (CAECE) dicta dos carreras tendientes a titulaciones diferentes: la Licenciatura en Psicología y la Licenciatura en Psicología Social, también un caso único. Ello totaliza 58 carreras de psicología de grado ofrecidas por las universidades de gestión privada.

Es decir, si se consideran las carreras de psicología que se dictan en universidades de gestión pública y en universidades de gestión privada, a agosto de 2014 puede verificarse la existencia en Argenti- na de un total de 70 carreras de grado de psicología (Tabla 5). De ellas 11 corresponden a carreras de universidades de gestión pública nacional, una a una carrera de una universidad de gestión pública provincial y 58 a universidades de gestión privada. Puede apreciarse que más del $80 \%$ de las carreras de psicología se ofrecen en el sistema privado, mientras solamente $17 \%$ en el sistema público. Llama la atención además, que 53 de esas carreras (cinco de universidades públicas y 48 de universidades privadas) son posteriores a 1990, esto es, tres cuartas partes de las carreras $(75,71 \%$ del total).

Hay que observar, no obstante, que ese predominio en la oferta educativa de carreras de psicología de gestión privada no se corresponde ni con el número total de graduados ni con el número de estudiantes. En efecto, según los últimos datos disponibles, sobre un total de 88290 graduados en 


\section{TABLA 4}

Universidades privadas que ofrecen carreras de Psicología (desde 1990)

\begin{tabular}{|c|c|c|c|}
\hline Universidad Privada & Inicio & Unidad Académica, sedes y lugar de emplazamiento & Título que otorga \\
\hline Universidad Adventista del Plata & 1990 & $\begin{array}{l}\text { Facultad de Humanidades, Educación y Ciencias Sociales. } \\
\text { Libertador San Martín. Entre Ríos }\end{array}$ & Lic. en Psicología \\
\hline Universidad de la Cuenca del Plata & 1994 & $\begin{array}{l}3 \text { sedes: 1) Corrientes, Fac. de Psicol., Educación y Rel. } \\
\text { Humanas, 2) Formosa, Fac. de Cs. Sociales y 3) Posadas, Fac. de } \\
\text { Cs. Sociales }\end{array}$ & Lic. en Psicología \\
\hline $\begin{array}{l}\text { Universidad Católica de Santiago del } \\
\text { Estero }\end{array}$ & 1995 & $\begin{array}{l}3 \text { sedes: Facultad de Ciencias de la Educación. Santiago del } \\
\text { Estero; Rafaela y San Salvador, Prov. Jujuy }\end{array}$ & Lic. en Psicología \\
\hline Universidad de Flores & 1995 & $\begin{array}{l}\text { Facultad de Psicología y Ciencias Sociales. } 2 \text { sedes, CABA y } \\
\text { Cipoletti }\end{array}$ & Lic. en Psicología \\
\hline Universidad Atlántida Argentina & 1995 & Fac. Psic. 3 sedes: Mar de Ajó, Dolores y Mar Plata & Lic. en Psicología \\
\hline Universidad Empresarial Siglo 21 & 1996 & $\begin{array}{l}2 \text { sedes Depto. de Psicología. Campus Córdoba y Campus Río } \\
\text { Cuarto }\end{array}$ & Lic. en Psicología \\
\hline Universidad Abierta Interamericana & 1997 & $\begin{array}{l}\text { Facultad de Psic y Relac. Humanas. } 5 \text { sedes: Buenos Aires, } \\
\text { Rosario, Ituzaingó, Berazategui; Lomas de Zamora * }\end{array}$ & Lic. en Psicología \\
\hline Universidad de la Marina Mercante & 1997 & Facultad de Humanidades. CABA & Lic. en Psicología \\
\hline Universidad de Palermo & 1998 & Facultad de Ciencias Sociales. CABA & Lic. en Psicología \\
\hline Universidad de Morón & 1998 & Fac. de Filosofía, Cs de la Educ. y Hum & Lic. en Psicología \\
\hline Universidad CAECE & $\begin{array}{l}1999 \\
2005\end{array}$ & Departamento de Psicología y Ciencias Pedagógiccas. CABA & $\begin{array}{l}\text { Lic. en Psic. Social } \\
\text { Lic. en Psicología }\end{array}$ \\
\hline $\begin{array}{l}\text { Universidad de Ciencias Empresariales } \\
\text { y Sociales (UCES) }\end{array}$ & 2000 & $\begin{array}{l}5 \text { sedes: Fac. de Psicología y Ciencias Sociales: Buenos Aires, } \\
\text { Rafaela, Olivos, San Isidro, Cañuelas ** }\end{array}$ & Lic. en Psicología \\
\hline Universidad Católica de Santa Fe & 2002 & Facultad de Psicología. Santa Fé & Lic. en Psicología \\
\hline Universidad Maimónides & 2002 & $\begin{array}{l}\text { Facultad de Humanidades, Ciencias Sociales y Empresariales. } \\
\text { CABA }\end{array}$ & Lic. en Psicología \\
\hline Universidad de Congreso & 2004 & Departamento de Psicología. Mendoza *** & Lic. en Psicología \\
\hline Universidad Argentina de la Empresa & 2004 & Facultad de Ciencias Jurídicas y Sociales. CABA & Lic. en Psicología \\
\hline Universidad de Mendoza & 2005 & Facultad de Ciencias de la Salud. 2 sedes: Mendoza y San Rafael & $\begin{array}{l}\text { Lic. en Psicología } \\
\text { con orientaciones }\end{array}$ \\
\hline $\begin{array}{l}\text { Instituto Universitario de Ciencias de } \\
\text { la Salud - Fund. Universitaria Barceló }\end{array}$ & 2006 & Facultad de Medicina. 2 sedes: Buenos Aires y La Rioja & Lic. en Psicología \\
\hline Universidad Favaloro & 2007 & Facultad de Ciencias Médicas. CABA & Lic. en Psicología \\
\hline Instituto Univ. Italiano de Rosario & 2007 & Escuela de Psicología. Rosario. Prov. Santa Fe & Lic. en Psicología \\
\hline
\end{tabular}

* En el proceso de acreditación, la sede de Ituzaingó se presentó como extensión áulica de la de Buenos Aires (Resolución CO. NEAU 1028 de 2013).

** En el proceso de acreditación, se presentaron únicamente 2 sedes, la de Buenos Aires y la de Rafaela (Resoluciones CO. NEAU 1018 y 1041 de 2013). Las restantes extensiones áulicas se iniciaron con posterioridad.

*** Entre 2006 y 2011 también se dictó la carrera en Córdoba, luego cerrada.

Fuente: relevamiento del autor con base en datos públicos de las propias universidades, comunicaciones personales y Ministerio de Educación, Secretaría de Políticas Universitarias, Dirección Nacional de Gestión Universitaria (2014). Base de títulos oficiales. Recuperado de http://titulosoficiales.siu.edu.ar/y resoluciones de CONEAU.

psicología entre 1960 y noviembre de 2014, 60412 graduados $(64.5 \%)$ obtuvieron su titulación de grado en universidades públicas y 332296 (35.5\%) en universidades privadas (Alonso \& Klinar, 2014). Por su parte, sobre un total de 74570 estudiantes de psi- cología en noviembre del 2014, 50340 estudiaban en universidades de gestión pública (67.5\%) y 24240 (32.5\%) en universidades de gestión privada (Alonso \& Klinar, 2014). En síntesis, no obstante que de cada 10 carreras de psicología, 8 corresponden a 
TABLA 5

Cantidad total de carreras de grado en psicología: públicas y privadas 2014

\begin{tabular}{|c|c|c|c|c|}
\hline & \multicolumn{2}{|c|}{ Universidades Públicas } & \multicolumn{2}{|c|}{ Universidades privadas } \\
\hline & $\begin{array}{c}\text { Cantidad de } \\
\text { Univ. }\end{array}$ & $\begin{array}{c}\text { Cantidad total } \\
\text { de carreras }\end{array}$ & $\begin{array}{l}\text { Cantidad de } \\
\text { Univ. }\end{array}$ & $\begin{array}{l}\text { Cantidad total } \\
\text { de carreras }\end{array}$ \\
\hline Universidades que dictan la carrera en una sola sede & 8 & 8 & 16 & 16 \\
\hline Universidades que dictan la carrera en 2 sedes & 2 & 4 & 6 & 12 \\
\hline Universidades que dictan la carrera en 3 sedes & - & - & 6 & 18 \\
\hline Universidades que dictan la carrera en 5 sedes & - & - & 2 & 10 \\
\hline Universidades que dictan 2 carreras con titulación diferente & - & - & 1 & 2 \\
\hline Totales para cada sistema (público o privado) & & $12(17.14 \%)$ & & $58(82.85 \%)$ \\
\hline Cantidad total de carreras en ambos sistemas & & & & \\
\hline
\end{tabular}

Fuente: relevamiento del autor en base a datos públicos de la propias universidades y en base a: Ministerio de Educación. Secretaría de Políticas Universitarias. Dirección Nacional de Gestión Universitaria (2014). Base de títulos oficiales.

Tabla 6

Cantidad de graduados y estudiantes de psicología en Argentina

\begin{tabular}{lcccc}
\hline & Cantidad de Estudiantes en noviembre 2012 & $\%$ & Cantidad de Graduados 1960-2012 & $\%$ \\
\hline Universidades públicas & 50.340 & 67.5 & 60.412 & 64.5 \\
Universidades privadas & 24.240 & 32.5 & 33.296 & 35.5 \\
Totales & 74.580 & 100 & 93.708 & 100 \\
\hline
\end{tabular}

Fuente: Alonso y Klinar (2014). Datos reconstruidos con su autorización.

universidades de gestión privada, casi dos tercios de los graduados han obtenido su título de grado en universidades de gestión pública y más de dos tercios de los estudiantes cursan en universidades de gestión pública y privada (Tabla 6).

En relación con la distribución geográfica de las carreras de psicología, el panorama también experimentó un cambio espectacular a partir de la década de 1990 y ha coincidido con ese fenómeno de "expansión, de diferenciación institucional y de distribución territorial" observado por Pérez Lindo (2007, p. 19). Hasta aquel momento, para realizar estudios de psicología había que trasladarse a algunas de las siguientes localidades: Ciudad de Buenos Aires, La Plata, Mar del Plata, Rosario, Córdoba, San Luis, Tucumán, Mendoza, San Juan o Salta. Tan solo en ocho provincias y Capital Federal se podían realizar estudios de grado de psicología.

En agosto de 2014, resulta posible realizar estudios de psicología en 15 provincias y en la Ciudad Autónoma de Buenos Aires. Solamente no es po- sible cursar carreras de psicología en las provincias de Catamarca, Chaco, Chubut, La Pampa, Neuquén, Santa Cruz y Tierra del Fuego (Tabla 7). Sin embargo, aun cuando no exista la carrera de psicología en Neuquén, la carrera de psicología de la Universidad del Comahue y una sede de la Universidad de Flores se encuentran en la sede de Cipoletti, en el Alto Valle del Río Negro, a menos de $10 \mathrm{~km}$ de la ciudad de Neuquén. Asimismo, en varias provincias existen ofertas curriculares en ciudades diferentes y en muchas grandes ciudades también varias carreras disponibles.

Sin embargo, casi 4 de cada 10 de las carreras se encuentran en la ciudad de Buenos Aires y exactamente la mitad de todas las carreras (35) están ubicadas en la Ciudad de Buenos Aires, Gran Buenos Aires y en la Provincia de Buenos Aires (50\%). Si a ese porcentaje se le agregan las carreras ubicadas en Santa Fé, Córdoba y Entre Rios, se constata que 50 carreras de psicología, casi las tres cuartas partes del total (71.42\%), están ubicadas en la zona conocida como "pampa húmeda", la zona más rica 
TABLA 7

Carreras de psicología: públicas y privadas. Distribución por ciudad y zona o provincia

\begin{tabular}{|c|c|c|c|c|}
\hline Ciudad & Total ciudad & Provincia o Zona & Total prov. o zona & $\%$ \\
\hline Ciudad Autónoma de Buenos Aires & 17 & \multirow{4}{*}{ Zona Metropolitana } & \multirow{4}{*}{ 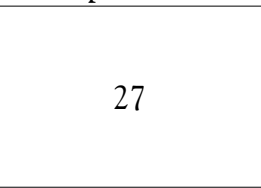 } & \multirow{4}{*}{38.57} \\
\hline Gran Buenos Aires Zona Norte & 4 & & & \\
\hline Gran Buenos Aires Zona Oeste & 2 & & & \\
\hline Gran Buenos Aires Zona Sur & 4 & & & \\
\hline La Plata & 2 & \multirow{6}{*}{ Prov. Buenos Aires } & \multirow{6}{*}{8} & \multirow{6}{*}{11.43} \\
\hline Mar del Plata & 2 & & & \\
\hline Mar de Ajó & 1 & & & \\
\hline Dolores & 1 & & & \\
\hline Chivilcoy & 1 & & & \\
\hline Bahía Blanca & 1 & & & \\
\hline Rosario & 4 & \multirow{3}{*}{ Prov. de Santa Fé } & \multirow{3}{*}{7} & \multirow{3}{*}{10} \\
\hline Rafaela & 2 & & & \\
\hline Santa Fé & 1 & & & \\
\hline Córdoba & 3 & \multirow{3}{*}{ Prov. de Córdoba } & \multirow{3}{*}{5} & \multirow{3}{*}{7.14} \\
\hline Río Cuarto & 1 & & & \\
\hline Marcos Juarez & 1 & & & \\
\hline Mendoza & 4 & \multirow{2}{*}{ Prov. de Mendoza } & \multirow{2}{*}{5} & \multirow{2}{*}{7.14} \\
\hline San Rafael & 1 & & & \\
\hline Paraná & 2 & \multirow[b]{2}{*}{ Prov. de Entre Ríos } & \multirow[b]{2}{*}{3} & \multirow{2}{*}{4.28} \\
\hline Libertador General San Martín & 1 & & & \\
\hline San Miguel de Tucumán & 2 & \multirow{2}{*}{ Prov. de Tucumán } & \multirow{2}{*}{3} & \multirow{2}{*}{4.28} \\
\hline Concepción & 1 & & & \\
\hline La Rioja & 2 & Prov. de La Rioja & 2 & 2.85 \\
\hline Cipoletti & 2 & Prov. de Río Negro & 2 & 2.85 \\
\hline Corrientes & 1 & Prov. de Corrientes & 1 & 1.42 \\
\hline Formosa & 1 & Prov. de Formosa & 1 & 1.42 \\
\hline Posadas & 1 & Prov. de Misiones & 1 & 1.42 \\
\hline San Salvador de Jujuy & 1 & Prov. De Jujuy & 1 & 1.42 \\
\hline Salta & 1 & Prov. De Salta & 1 & 1.42 \\
\hline San Juan & 1 & Prov. de San Juan & 1 & 1.42 \\
\hline San Luis & 1 & Prov. de San Luis & 1 & 1.42 \\
\hline Santiago del Estero & 1 & Prov. de Sgo. Del Estero & 1 & 1.42 \\
\hline Totales & 70 & & 70 & 99.9 \\
\hline
\end{tabular}

y poblada del país, integrada por la Ciudad Autónoma de Buenos Aires, Provincia de Buenos Aires, Córdoba, Santa Fé y Entre Ríos.

El estudio de Modesto Alonso y Doménica Klinar (2014), con datos correspondientes a 2013, indicaban que del total de 93708 graduados en psicología en todo el país desde 1960, 37688 se habían graduado en la Universidad de Buenos Aires (40.21\%). Es decir, que de cada 10 psicólogos o psicólogas en el país, 4 se recibieron en la Universidad de Buenos. Pero considerando la Universidad Nacional de La Plata y las universidades privadas de la zona metropolitana, el total de egresados de esa zona asciende a 71 281, es decir el $76.06 \%$ de graduados y graduadas en psicología en todo el país (Alonso \& Klinar, 2014). Si se amplían esos datos con los provenientes de las restantes universidades ubicadas en la denominada "pampa húmeda", es 
TABLA 8

Comparación del porcentaje de distribución geográfica en la pampa húmeda de las carreras de psicología con otros indicadores

\begin{tabular}{lcc}
\hline & Cantidad & \% sobre la población total \\
\hline Carreras de Psicología en la zona de la pampa húmeda & 50 & 71.42 \\
Cantidad de psicólogos/as activos/as en la pampa húmeda * & 85.397 & 92.04 \\
Población total en la pampa húmeda ** & 26.254 .642 & 65.45 \\
Profesionales en CABA, Prov. de Bs. As. y de Santa Fé *** & 606.802 & 70.7 \\
Estudiantes internacionales en la región metropolitana y bonaerense **** & 19.985 & 75.83 \\
\hline
\end{tabular}

Fuentes: * Alonso y Klinar (2014)

** INDEC (2012)

*** INDEC (2005)

**** Ministerio de Educación. Secretaría de Políticas Universitarias (2007)

decir la zona más rica del país, el porcentaje de graduados supera el $90 \%$ (Tabla 8). Es decir, la distribución geográfica de las carreras de psicología y de los graduados en psicología resulta consistente con la distribución geográfica del conjunto de profesionales en el país, aunque la concentración de psicólogos y psicólogas en la zona de la pampa húmeda es todavía mayor.

Un importante punto de inflexión en la enseñanza universitaria de la psicología en Argentina se produjo con la inclusión de la titulación de psicología entre las actividades incluidas en la Ley de Educación Superior (LES), que fue dictada durante el gobierno de Carlos Menem en una situación de máxima conflictividad con el movimiento estudiantil y en general con los sectores más críticos del profesorado universitario. El art. 43 de la mencionada ley establecía:

Cuando se trate de títulos correspondientes a profesionales reguladas por el Estado, cuyo ejercicio pudiera comprometer el interés público poniendo en riesgo de modo directo la salud, la seguridad, los derechos, los bienes o la formación de los habitantes, se requerirá que se respeten, además de la carga horaria a la que hace referencia el artículo anterior, los siguientes requisitos:

a) Los planes de estudio deberán tener en cuenta los contenidos curriculares básicos y los criterios sobre intensidad de la formación práctica que establezca el Ministerio de Educación, en acuerdo con el Consejo de Universidades; b) Las carreras respectivas deberán ser acreditadas periódicamente por la Comisión Nacional de Evaluación y Acreditación Universitaria o por entidades privadas constituidas con ese fin debidamente reconocidas.

El Ministerio de Educación determinará con criterio restrictivo, en acuerdo con el Consejo de universidades, la nómina de tales títulos, así como las actividades profesionales reservadas exclusivamente para ellos. (Ley de Educación Superior, 1995, p. 3)

La Ley de Educación Superior dejaba a criterio del Consejo de Universidades, compuesto por representantes de universidades públicas y de universidades privadas, cuáles serían las titulaciones reguladas por el Estado.

Al mismo tiempo, la ley también creaba el organismo encargado del proceso de acreditación, tanto para los programas de grado, que en el caso de aquellos comprendidos en el art. 43 pasaban a ser obligatorios como para los de posgrado, cuya acreditación era relativamente voluntaria: la Comisión Nacional de Evaluación y Acreditación Universitaria (CONEAU), concebido como un organismo descentralizado y autónomo en esfera del Ministerio de Educación.

En ese marco, la Federación de Psicólogos de la República Argentina solicitó al Ministerio de Educación la inclusión del título de psicólogo y licenciado en psicología (a los efectos legales en Argentina se los considera sinónimos) entre aquellos comprendidos por la ley, de manera de garantizar 
el reconocimiento de las actividades reservados al título de psicólogo o licenciado en psicología. Un año después la Asociación de Unidades Académicas (AUAPsi), que nuclea a las carreras de psicología de universidades de gestión pública, realizó una petición en la misma dirección y comenzó a elaborar criterios para la acreditación. A su vez, también la Unidad de Vinculación Académica de Psicología de Universidades de Gestión Privada (UVAPsi), que nuclea a las carreras de psicología de gestión privada, comenzó su propio proceso de elaboración de los estándares para la acreditación (Di Doménico \& Piacente, 2003, 2011; Di Doménico \& Risueño, 2013).

En marzo de 2004, el Ministerio de Educación, Ciencia y Tecnología aprobó la Resolución 136 que en su artículo primero declaraba "incluido al título de Licenciado en Psicología en el régimen del artículo 43 de la Ley № 24.521" (Ministerio de Educación, Ciencia y Tecnología, 2004, p. 4). Para continuar el proceso que debía establecer los estándares para la evaluación y acreditación de las carreras de grado, el Ministerio solicitó a AUAPsi y UVAPsi que los elaboraran de manera conjunta.

Finalmente, en septiembre de 2009 establecían los criterios para el proceso de acreditación de carreras de grado de psicología a través de la Resolución ministerial 343/09 (Ministerio de Educación, 2009).

Artículo 1ํㅡ- Aprobar los contenidos curriculares básicos, la carga horaria mínima, los criterios de intensidad de la formación práctica y los estándares para la acreditación de las carreras correspondientes a los títulos de PSICOLOGO y LICENCIADO EN PSICOLOGIA, así como la nómina de actividades reservadas para quienes hayan obtenido los títulos respectivos, que obran como Anexos I — Contenidos Curriculares Básicos—, II —Carga Horaria Mínima-, III —Criterios de Intensidad de la Formación Práctica_, IV — Estándares para la Acreditacióny V -Actividades Profesionales Reservadas - de la presente resolución. (Ministerio de Educación, 2009, p. 7)

A lo largo de los cinco años que mediaron entre la resolución 136/04 y la resolución 343/09, la AUAP. si, la UVAPsi e inclusive la Federación de Psicólogos de la República Argentina (FePRA) participaron de un intenso debate acerca del perfil del graduado en psicología, de las actividades correspondientes a tal titulación y de los contenidos que posibilitaran una formación adecuada para el desarrollo de tales actividades (Benito, 2009; de la Cruz, 2010; Di Doménico, en prensa; Di Doménico \& Piacente, 2003, 2011; Di Doménico \& Risueño, 2013; Ferrero $\&$ de Andrea, 2011; Moya, 2010). En ese sentido, puede afirmarse que la resolución 343/09 con sus fortalezas, limitaciones y debilidades es el resultado del consenso alcanzado y del grado de avance de la psicología académica y profesional en argentina al finalizar la primera década del siglo XXI.

El diseño curricular contemplado en la organización del plan de estudios en la resolución 343 y su modificación parcial, la Resolución 800/11 (Ministerio de Educación, 2011), no se estructuró a partir del perfil del graduado ni de las habilidades o competencias o calificaciones que el futuro graduado debía exhibir, sino que se estructuró en función de contenidos o áreas de conocimiento:

A. Procesos biopsicosociales

B. Desarrollos de la psicología en relación con los individuos, parejas, familias, grupos, instituciones, organizaciones y comunidades.

C. Procesos psicopatológicos

D. Historia de la psicología

E. Metodología de la investigación psicológica

F. Evaluación y diagnóstico psicológicos

G. Intervenciones en psicología

H. Formación complementaria en otras disciplinas

I. Ética y deontología profesional. (Ministerio de Educación, 2009, p. 8)

Otro aspecto de la formación que la Resolución 343 y su modificatoria parcial, la Resolución 800/11 destacaron, fue la definición de la carga horaria mínima del plan de estudios y la carga horaria práctica, tanto de las prácticas habituales en las asignaturas o cursos como las prácticas profesionales tutoriadas que en la modificación parcial de la resolución fueron denominadas prácticas profesionales supervisadas (Ministerio de Educación, 2011, p. 13): 
Se define una carga horaria total mínima de tres mil doscientas (3200) horas, áulicas y extraáulicas. El número de horas con presencia simultánea de docentes y alumnos no podrá ser inferior a 2600. Las horas podrán ser teóricas, teórico-prácticas, prácticas supervisadas, prácticas pre-profesionales. De la carga horaria total, dos mil setecientas horas (2700) se destinarán a la formación teórica y las restantes quinientas (500) se referirán a la formación práctica. (Ministerio de Educación, 2011, p. 13)

Las horas prácticas serán de 2 tipos:

a) Horas prácticas que formarán parte de las asignaturas con indicadores visibles en los respectivos programas y que proporcionarán productos tangibles tales como: monografías, informes escritos, observaciones, entrevistas, encuestas, sondeos de opinión, trabajos de evaluación psicológica, estudio de casos, investigación, trabajos de campo. Las mismas estarán destinadas a la adquisición de habilidades y conocimientos metodológicos y de dispositivos de evaluación e intervención psicológicas.

b) Horas prácticas profesionales supervisadas (PPS) tendrán una carga horaria mínima de doscientas cincuenta horas (250). Los objetivos y características principales de estas prácticas intensivas e integrativas, son la vinculación del mundo académico con el mundo del trabajo, a través de la integración de los conocimientos teórico-prácticos, que garanticen el aprendizaje de los contenidos procedimentales ("saber hacer") y de las reglas de funcionamiento profesional. Deben implementarse en el último tramo del trayecto formativo, cuando el alumno esté en condiciones de contar con los conocimientos que las posibilitan. Para ello, los estudiantes se incorporan a proyectos o programas de la propia unidad académica o de instituciones u organizaciones en las que se desempeñan profesionales de la disciplina en posiciones laborales específicas (incluida la de investigación). (Ministerio de Educación, 2011, p. 13)

La inclusión de las horas prácticas, la caracterización y clasificación de la misma, tanto como los criterios para la intensidad que también incorpora la normativa y la inclusión de un Trabajo Integra- dor Final obligatorio (TIF), constituyeron seguramente los aspectos más novedosos para el sistema. Al mismo tiempo, fundamentaron buena parte de las polémicas del proceso de acreditación abierto en 2012, que posibilitó las primeras resoluciones por parte de la CONEAU en diciembre de 2013, pero que jurídicamente y académicamente aun no ha finalizado, ya que las carreras que no fueron acreditadas tenían plazo para presentar recursos de reconsideración hasta finales de 2014.

La Resolución 343 también estableció los restantes estándares para la acreditación, cuyas dimensiones de análisis no diferían de los que se venían aplicando para la acreditación de las carreras de posgrado: contexto institucional, formación y plan de estudios, cuerpo académico, estudiantes y graduados, personal de apoyo, infraestructura, equipamiento y recursos presupuestarios (Ministerio de Educación, 2009).

Dichas dimensiones de análisis incorporaban datos relacionadas con el cuerpo académico, los estudiantes, graduados y el personal de apoyo, indicadores que ya habían sido analizados en el diagnóstico encarado por la AUAPsi en el período 1996-1999 (AUAPsi, 1998). Inclusive, el apartado de los estándares relacionados con infraestructura, equipamientos y recursos presupuestarios, retrocedía respecto a indicadores que AUAPsi había considerado, y ponía de manifiesto importantes limitaciones de la gestión acreditadora. Por ejemplo, la resolución establecía que la "carrera debe tener acceso a bibliotecas y centros de información actualizados que dispongan de un acervo bibliográfico pertinente y variado" (Ministerio de Educación, 2009, p. 10). De esa manera, se excluía del análisis al acceso de estudiantes y docentes a hemerotecas y a bases de datos especializadas para la disciplina (PsycInfo, Psicodoc, ERIC, Pubmed, Sociological Abstracts) y repertorios a textos completos, que finalmente constituyen la garantía más segura para la actualización permanente del conocimiento (Visca, 2010).

Esa omisión llama más la atención si se considera que la Biblioteca Electrónica de Ciencia y Tecnología, la cual incluye buena parte de esas bases de datos, ha constituido una iniciativa oficial, que 
por lo mismo el Ministerio de Educación no podía desconocer. Y sobre todo, si se considera que también desde la Secretaría de Ciencia, Tecnología e Innovación Productiva de la Nación se venía impulsando desde varios años atrás una ley de repositorios públicos de ciencia y técnica, que obligaba a las instituciones generadoras de producción de conocimiento con financiamiento público a generar repositorios abiertos y gratuitos que garantizaran la libre circulación de los productos de investigación (Bongiovani \& Nakano, 2011).

Es decir, por una parte, la resolución ministerial acordada por AUAPsi y UVAPsi evidenciaba el estado actual de avance de los académicos de la disciplina, el cual no parece ser el más actualizado. Por otra, también pone de manifiesto la relativa desactualización de algunas instancias técnicas intermedias, tanto en Ministerio de Educación como en la CONEAU.

Con todo, el proceso de acreditación tuvo lugar a lo largo del año 2012 y las resoluciones de la CONEAU se emitieron a finales del año 2013. El resultado, hasta agosto de 2014, es que de las 70 carreras se han acreditado únicamente 28 carreras. Dejando de lado el caso de las carreras que no se presentaron a acreditar porque su titulación no estaba incluida en la convocatoria (el caso de la Licenciatura en Psicología Organizacional y en Psicología Social), tampoco se presentó a acreditar la carrera de psicología de la Universidad de Buenos Aires por considerar que la Ley de Educación Superior, que introduce la acreditación, vulnera la autonomía universitaria. En cualquier caso, el resultado final todavía es incierto porque las universidades que no han acreditado hasta la fecha han presentado recursos de reconsideración que, posiblemente, en algunos casos puedan terminar dirimiéndose en instancias judiciales por dos razones principales. La primera, por la complejidad del derecho administrativo argentino; la segunda, porque se han observado varias inconsistencias en los dictámenes emitidos por la CONEAU sobre un mismo tipo de déficit con un mismo tipo de plan de mejoramiento, pero que era considerado insalvable para algunos casos mientras que se consideraba adecuado para otros.
Es interesante observar que las resoluciones aprobadas por la CONEAU de acreditación por 6 años, que supone el cumplimiento de todos los estándares, han recaído en solamente 6 universidades privadas, algunas de ellas con una larga tradición en la enseñanza de la psicología en el país (como la Universidad del Salvador o la Universidad del Aconcagua), otras que ya habían acreditado carreras de doctorado con un alto nivel académico (Universidad de Palermo). Pero también se encuentran allí carreras muy recientes y que no se encuentran entre aquellas carreras de psicología que han jerarquizado ciertos rankings internacionales, como el Ranking Iberoamericano de Psicología elaborado por Scimago Research Group (2011). Es verdad que el sistema de acreditación de carreras de grado que establece la ley argentina no propone un ranking sino el cumplimiento total o parcial de los estándares para la carrera en cuestión. Pero en una primera lectura de las resoluciones publicadas en la página de la CONEAU, llaman la atención ciertas discordancias entre la evaluación de la CO. NEAU y la realizada, por ejemplo, por Scimago. No queda claro si ello se debe a que Scimago toma en consideración cuatro indicadores relacionados publicaciones científicas, a la falta de actualización ya apuntada de los indicadores que la CONEAU evaluaba, o a la dinámica evidenciada por las universidades privadas que, una vez aprobada la Resolución 343/09, en poco tiempo modificaron sus planes de estudios y sus reglamentaciones internas para adecuarlas a la misma.

Por su parte, el sistema de posgrado en psicología es relativamente incipiente. Por lo pronto, desde el punto de vista legal, las titulaciones de grado habilitan para el ejercicio de la profesión sin ningún tipo de restricciones. Más todavía, la titulación de posgrado tampoco es requerimiento para el ejercicio de la profesión académica, salvo para la carrera de investigador científica del Consejo Nacional de Investigaciones Científicas y Técnicas (CONICET).

Nuevamente, no resulta sencillo precisar todas las carreras de posgrado en psicología existentes en el país. Aquí hemos relevado todas aquellas acreditadas por la CONEAU, sabiendo que algunas de ellas no abren su inscripción todos los años o que 
otras pueden haberse cerrado definitivamente. Por otro lado, también se verifica la existencia de carreras de posgrado que no han sido acreditadas por la CONEAU. La CONEAU reconoce 3 tipos de carreras de posgrado: los doctorados, las maestrías (que a su vez pueden ser académicas o profesionales) y las carreras de especialización (CONEAU, 2011).

En primer lugar, puede observarse el débil desarrollo de la psicología como disciplina académica. En efecto, existen únicamente 14 carreras de doctorado, una sola acreditada con la más alta categoría, la categoría A (Tabla 9). Por su parte, existen 39 carreras de Maestría, otra vez, una sola acreditada A (Tabla 10) y 33 carreras de especialización, ninguna acreditada A (Tabla 11). Es decir, de las 86 carreras de posgrado acreditadas por CONEAU, solamente 2 reúnen los más altos niveles académicos $(2.32 \%$ del total). Por su parte, solamente 15 carreras de posgrado alcanzaron la categoría B, apenas el $17.44 \%$.
Todo ello refleja el desarrollo de la psicología argentina, fuertemente sesgado hacia la práctica profesional, especialmente en el campo de la clínica o la psicoterapia. 16 posgrados están orientados a la clínica o psicoterapia $(18.6 \%)$ y un total de 20 son explícitamente sobre psicoanálisis (23\%), aun cuando el psicoanálisis atraviesa otras carreras de posgrado también. Si a esos programas agregamos las carreras de posgrado centradas en el psicodiagnóstico y la evaluación, que en Argentina también ha estado orientada por la clínica, se advierte que alrededor de la mitad de los programas de posgrado se centran en la enseñanza de la clínica.

Ello no puede sorprender; ya en uno de los primeros estudios sobre la profesión del psicólogo en Argentina, se concluía que el 77 \% de psicólogos y psicólogas en la ciudad de Buenos Aires trabajaban en el área clínica (Litvinoff \& Gomel, 1973). Estimaciones contemporáneas indican que aproximadamente entre el 40 y el $90 \%$ se dedican a la

TABLA 9

Posgrados en Psicología - Doctorados acreditados por la CONEAU

\begin{tabular}{|c|c|c|c|}
\hline Carrera de Posgrado & Universidad & $\begin{array}{l}\text { CONEAU } \\
\text { Acredit. }\end{array}$ & Categ. \\
\hline Doctorado en Psicología & Universidad Nacional de Córdoba & $295 / 07$ & A \\
\hline Doctorado en Psicología & Universidad Nacional de La Plata & $491 / 07$ & B \\
\hline Doctorado en Psicología & Universidad Nacional de San Luis & $294 / 07$ & B \\
\hline $\begin{array}{l}\text { Doctorado de la Universidad de Palermo en } \\
\text { Psicología }\end{array}$ & Universidad de Palermo & $427 / 07$ & B \\
\hline $\begin{array}{l}\text { Doctorado de la Universidad de Buenos } \\
\text { Aires, Facultad de Psicología }\end{array}$ & Universidad de Buenos Aires & $448 / 13$ & B \\
\hline Doctorado en Psicología & Universidad Nacional de Mar del Plata & $447 / 13$ & B \\
\hline Doctorado en Psicología & Universidad de Ciencias Empresariales y Sociales & $1180 / 13$ & B \\
\hline Doctorado en Psicología & Universidad Nacional de Rosario & $592 / 07$ & $\mathrm{C}$ \\
\hline Doctorado en Psicología Social & Universidad Argentina John F. Kennedy & $204 / 08$ & $\mathrm{C}$ \\
\hline Doctorado en Psicología & Universidad Nacional de Tucumán & $484 / 04$ & $\mathrm{Cn} *$ \\
\hline Doctorado en Psicología & Universidad del Salvador & $446 / 13$ & No sol **: \\
\hline $\begin{array}{l}\text { Doctorado en Psicología con mención en } \\
\text { Integración Cognitivo - Existencial }\end{array}$ & Universidad de Flores & $638 / 05$ & No sol \\
\hline Doctorado en Psicología & Universidad Católica Argentina & $445 / 13$ & No sol. \\
\hline $\begin{array}{l}\text { Doctorado de la Universidad Maimónides } \\
\text { en Psicología con orientación en } \\
\text { Neurociencia Cognitiva Aplicada }\end{array}$ & Universidad Maimónides & $484 / 05$ & Proy $* * *$ \\
\hline
\end{tabular}

* La categorización B o C seguida de n, signifca que era una carrera nueva al momento de la acreditación.

** No sol: no solicitó categorización.

*** es un proyecto de carrera.

Fuente: CONEAU (2014) 
TAbla 10

Posgrados en Psicología - Maestrías acreditadas por la CONEAU

\begin{tabular}{|c|c|c|c|}
\hline Carrera de Posgrado & Universidad & $\begin{array}{l}\text { CONEAU } \\
\text { Acredit }\end{array}$ & Categ. \\
\hline Maestría en Psicoinmunoneuroendocrinología & Universidad Favaloro & $582 / 09$ & A \\
\hline Maestría en Psicodiagnóstico y Evaluación Psicológica & Universidad de Buenos Aires & $503 / 13$ & B \\
\hline Maestría en Psicología Cognitiva y Aprendizaje & Facultad Latinoamericana de Ciencias Sociales (FLACSO) & $470 / 07$ & B \\
\hline Maestría en Psicología Educacional & Universidad Nacional de Tucumán & $150 / 09$ & B \\
\hline Maestría en Psicología Educacional & Universidad de Buenos Aires & $442 / 14$ & B \\
\hline Maestría en Neuropsicología & Universidad Nacional de Córdoba & 280/01 & $\mathrm{Bn}$ \\
\hline Maestría en Psicoanálisis & Universidad del Aconcagua & 289/07 & $\mathrm{C}$ \\
\hline Maestría en Psicoterapia Sistémica & Universidad del Aconcagua & 288/07 & $\mathrm{C}$ \\
\hline Maestría en Psicoanálisis & Universidad Nacional de Rosario & $246 / 14$ & $\mathrm{C}$ \\
\hline Maestría en Psicoanálisis & Universidad Nacional de Mar del Plata & $474 / 08$ & $\mathrm{C}$ \\
\hline Maestría en Psicoanálisis & Universidad Argentina John F. Kennedy & $590 / 07$ & $\mathrm{C}$ \\
\hline Maestría en Psicoanálisis Teórico & Universidad Nacional de San Luis & $383 / 00$ & $\mathrm{C}$ \\
\hline $\begin{array}{l}\text { Maestría en Fundamentos Teóricos de la Clínica } \\
\text { Psicoanalítica Lacaniana }\end{array}$ & Universidad Nacional de San Luis & $319 / 01$ & $\mathrm{C}$ \\
\hline $\begin{array}{l}\text { Maestría en Psicología Clínica Mención Cognitivo- } \\
\text { Integrativa }\end{array}$ & Universidad Nacional de San Luis & $530 / 13$ & C \\
\hline Maestría en Psicología Cognitiva & Universidad de Buenos Aires & $484 / 08$ & $\mathrm{C}$ \\
\hline Maestría en Psicología Social & Universidad Nacional de Cuyo & $531 / 13$ & $\mathrm{C}$ \\
\hline $\begin{array}{l}\text { Maestría en Psicología Social (Orientación, Grupos e } \\
\text { Instituciones) }\end{array}$ & Universidad Nacional de Tucumán & $444 / 04$ & $\mathrm{C}$ \\
\hline Maestría en Psiconeurofarmacología & Universidad Favaloro & $583 / 09$ & $\mathrm{C}$ \\
\hline Maestría en Psicooncología & Universidad Favaloro & $1146 / 13$ & $\mathrm{C}$ \\
\hline Maestría en Psicoanálisis & Universidad del Salvador & $507 / 07$ & $\mathrm{Cn}$ \\
\hline Maestría en Psicoanálisis & Universidad de Buenos Aires & $443 / 04$ & $\mathrm{Cn}$ \\
\hline Maestría en Psicología del Aprendizaje & Universidad del COMHUE & 298/99 & $\mathrm{Cn}$ \\
\hline Maestría en Psicología Preventiva & Universidad Nacional de Catamarca & $641 / 99$ & $\mathrm{Cn}$ \\
\hline Maestría en Psicología Social & Universidad Nacional de Mar del Plata & $865 / 99$ & $\mathrm{Cn}$ \\
\hline Maestría en Clínica Psicológica Cognitiva & Universidad de Belgrano & $502 / 13$ & No sol \\
\hline Maestría en Criminología & Universidad del Aconcagua & $598 / 07$ & $\mathrm{C}$ \\
\hline Maestría en Criminología & Universidad Nacional de Lomas de Zamora & $222 / 06$ & No sol \\
\hline Maestría en Cultura y Salud Mental & $\begin{array}{l}\text { Instituto Universitario de Salud Mental de la Asociación } \\
\text { Psicoanalítica de Bs. As. }\end{array}$ & $804 / 13$ & No sol \\
\hline Maestría en Familia y Minoridad & Universidad Católica de Cuyo & $957 / 99$ & No sol \\
\hline Maestría en Psicoanálisis & Universidad Nacional de La Matanza & $461 / 13$ & No sol \\
\hline Maestría en Psicología Social Comunitaria & Universidad de Buenos Aires & $462 / 13$ & No sol \\
\hline Maestría en Psicología Clínica & Universidad Empresarial Siglo 21 & 293/07 & No sol \\
\hline Maestría en Psic. Empresarial y Organizacional & Universidad de Belgrano & $243 / 09$ & No sol \\
\hline $\begin{array}{l}\text { Maestría en Psicología Organizacional con } \\
\text { Orientación Gerencial }\end{array}$ & Universidad Abierta Interamericana & $460 / 13$ & No sol \\
\hline Maestría en Familia y Discapacidad & Universidad del Museo Social Argentino & $065 / 03$ & Proy \\
\hline Maestría en Neuropsicología & Instituto Universitario Escuela de Medicina del Hospital Italiano & $395 / 05$ & Proy \\
\hline Maestría en Psicología de la Música & Universidad Nacional de La Plata & 299/06 & Proy \\
\hline Maestría en Psicología Social & Universidad de la Marina Mercante & $486 / 05$ & Proy \\
\hline Maestría en Psicología y Salud & Universidad de Palermo & $265 / 06$ & Proy \\
\hline
\end{tabular}

Fuente: CONEAU (2014) 


\section{TABLA 11}

Posgrados en Psicología - Carreras de especialización acreditadas por la CONEAU

\begin{tabular}{|c|c|c|c|}
\hline Carrera de Posgrado & Universidad & $\begin{array}{l}\text { CONEAU } \\
\text { Acred. }\end{array}$ & Categ \\
\hline Especialización en Psicodiagnóstico & Universidad Nacional de Rosario & $529 / 13$ & B \\
\hline $\begin{array}{l}\text { Especialización en Evaluación y Diagnóstico } \\
\text { Psicológico }\end{array}$ & Universidad Nacional de Tucumán & $201 / 14$ & B \\
\hline Especialización en Neuropsicología Clínica & Universidad de Buenos Aires & $497 / 13$ & B \\
\hline $\begin{array}{l}\text { Especialización en Psicología del Trabajo y de las } \\
\text { Organizaciones }\end{array}$ & Universidad Nacional de Córdoba & $527 / 13$ & B \\
\hline $\begin{array}{l}\text { Especialización en Psicología Clínica, Institucional } \\
\text { y Comunitaria }\end{array}$ & Universidad Nacional de Rosario & $1181 / 13$ & $\mathrm{C}$ \\
\hline $\begin{array}{l}\text { Especialización en Clínica Psicoanalítica con } \\
\text { Niños y Adolescentes }\end{array}$ & Universidad Nacional de La Plata & $1174 / 13$ & $\mathrm{C}$ \\
\hline $\begin{array}{l}\text { Especialización en Evaluación y Diagnóstico } \\
\text { Psicológico }\end{array}$ & Universidad Nacional de La Plata & $602 / 13$ & $\mathrm{C}$ \\
\hline $\begin{array}{l}\text { Especialización en Psicología Educacional con } \\
\text { orientación en procesos de aprendizaje del } \\
\text { lenguaje escrito y sus trastornos }\end{array}$ & Universidad Nacional de La Plata & $1185 / 13$ & $\mathrm{C}$ \\
\hline $\begin{array}{l}\text { Especialización en Clínica Psicoanalítica con } \\
\text { Adultos }\end{array}$ & Universidad Nacional de La Plata & $1173 / 13$ & $\mathrm{C}$ \\
\hline $\begin{array}{l}\text { Especialización en Evaluación y Diagnostico } \\
\text { Psicológico }\end{array}$ & Universidad del Salvador & $486 / 08$ & $\mathrm{C}$ \\
\hline $\begin{array}{l}\text { Especialización en orientación educativa y } \\
\text { ocupacional }\end{array}$ & Universidad Nacional de La Plata & $603 / 13$ & $\mathrm{C}$ \\
\hline $\begin{array}{l}\text { Especialización en Psicología Clínica con } \\
\text { Orientación Psicoanalítica }\end{array}$ & Universidad de Buenos Aires & $454 / 13$ & $\mathrm{C}$ \\
\hline Especialización en Psicología Clínica & Universidad Argentina John F. Kennedy & $420 / 07$ & $\mathrm{C}$ \\
\hline Especialización en Psicología en Educación & Universidad Nacional de Rosario & $141 / 07$ & $\mathrm{C}$ \\
\hline Especialización en Violencia Familiar & Universidad de Buenos Aires & $502 / 07$ & $\mathrm{C}$ \\
\hline $\begin{array}{l}\text { Especialización en Política y Gestión de la Salud } \\
\text { Mental }\end{array}$ & Universidad de Buenos Aires & $408 / 04$ & $\mathrm{Cn}$ \\
\hline $\begin{array}{l}\text { Especialización en Psicología Clínica de Niños y } \\
\text { Adolescentes (Orientación Psicoanalítica) }\end{array}$ & Universidad Nacional de Tucumán & $496 / 13$ & $\mathrm{Cn}$ \\
\hline Especialización en Evaluación Psicológica & dad de Buenos Aires & $498 / 13$ & No sol \\
\hline Especialización en Fundamentos de Psicoanálisis & Universidad Cuenca del Plata & $451 / 13$ & No sol \\
\hline Especialización en Psicoanálisis & $\begin{array}{l}\text { Instituto Universitario de Salud Mental de } \\
\text { la Asociación Psicoanalítica de Bs. As. }\end{array}$ & $456 / 13$ & No sol \\
\hline Especialización en Psicoanálisis con Adolescentes & $\begin{array}{l}\text { Universidad de Ciencias Empresariales y } \\
\text { Sociales }\end{array}$ & $457 / 13$ & No sol \\
\hline Especialización en Psicoanálisis con Niños & $\begin{array}{l}\text { Universidad de Ciencias Empresariales y } \\
\text { Sociales }\end{array}$ & $422 / 07$ & No sol \\
\hline $\begin{array}{l}\text { Especialización en Psicoanálisis con orientación } \\
\text { clínica en Adultos }\end{array}$ & Universidad Nacional de La Matanza & $42 / 14$ & No sol \\
\hline Especialización en Psicogerontología & Universidad Maimónides & $434 / 14$ & No sol \\
\hline $\begin{array}{l}\text { Especialización en Psicología Clínica Asistencial } \\
\text { Infantil }\end{array}$ & Universidad Católica de Cuyo & $887 / 05$ & No sol \\
\hline $\begin{array}{l}\text { Especialización en Psicología Clínica de Niños y } \\
\text { Adolescentes }\end{array}$ & $\begin{array}{l}\text { Instituto Universitario de Salud Mental de } \\
\text { la Asociación Psicoanalítica de Bs. As. }\end{array}$ & $455 / 13$ & No sol \\
\hline Especialización en Psicología Forense & $\begin{array}{l}\text { Universidad de Ciencias Empresariales y } \\
\text { Sociales }\end{array}$ & $423 / 07$ & No sol \\
\hline Especialización en Psicooncología & $\begin{array}{l}\text { Universidad de Ciencias Empresariales y } \\
\text { Sociales }\end{array}$ & $203 / 14$ & No sol \\
\hline
\end{tabular}




\begin{tabular}{|c|c|c|c|}
\hline Carrera de Posgrado & Universidad & $\begin{array}{l}\text { CONEAU } \\
\text { Acred. }\end{array}$ & Categ \\
\hline Especialización en Psicopatología y Salud Mental & $\begin{array}{l}\text { Instituto Universitario de Salud Mental de } \\
\text { la Asociación Psicoanalítica de Bs. As. }\end{array}$ & $840 / 13$ & No sol \\
\hline $\begin{array}{l}\text { Especialización en Psicoterapia Familiar con } \\
\text { Orientación Cognitiva }\end{array}$ & Universidad Maimónides & $504 / 07$ & No sol \\
\hline $\begin{array}{l}\text { Especialización en Psicoterapia Individual y } \\
\text { Grupal }\end{array}$ & Universidad Maimónides & $505 / 07$ & No sol \\
\hline $\begin{array}{l}\text { Especialización en Abordaje de Patologías } \\
\text { Psicosomáticas }\end{array}$ & Universidad CAECE & $002 / 03$ & Proy \\
\hline $\begin{array}{l}\text { Especialización en Abordaje Psicoanalítico en } \\
\text { Familia y Pareja }\end{array}$ & Universidad CAECE & $129 / 02$ & Proy \\
\hline
\end{tabular}

Fuente: CONEAU, 2014

clínica (Alonso \& Klinar, 2014), mientras menos del $1 \%$ lo hace, en el área comunitaria.

En los últimos quince o veinte años, la clínica de orientación cognitiva integrativa ha generado un interés creciente tanto entre profesionales como entre estudiantes de psicología. No obstante, en 2005 una de las dos instituciones psicoanalíticas argenti- nas adheridas a la International Psychoanalyticasl Association, la Asociación Psicoanalítica de Buenos Aires, logró el reconocimiento por parte de las autoridades argentinas a su Instituto Universitario de Salud Mental de la Asociación Psicoanalítica de Buenos Aires. Es decir, la psicología argentina de hoy, aun cuando ha experimentado una mayor

TABLA 12

Principales Organizaciones de la Psicología en Argentina

\begin{tabular}{|c|c|c|}
\hline Comienzo & Cierre & Institución \\
\hline \multicolumn{3}{|r|}{ Sociedades científicas en el campo de la psicología } \\
\hline 1908 & 1914 & Sociedad de Psicología \\
\hline 1930 & - & Sociedad de Psicología de Buenos Aires (desde 1956: Sociedad Argentina de Psicología) * \\
\hline 1942 & - & Asociación Psicoanalítica Argentina \\
\hline 1987 & & $\begin{array}{l}\text { Asociación Argentina de Ciencias del Comportamiento (AACC). Desde 1994, Miembro } \\
\text { Nacional por Argentina en IUPsyS }\end{array}$ \\
\hline 1988 & - & Asociación Argentina de Estudio e Investigación en Psicodiagnóstico (ADEIP) \\
\hline 2005 & - & Asociación para el Avance de la Ciencia Psicológica (AACP) \\
\hline \multicolumn{3}{|r|}{ Primeras instituciones integradas por egresados/as en carreras de Psicología } \\
\hline 7 Sept 1962 & 1985 & Asociación de Psicólogos de Rosario ** \\
\hline Nov 1962 & - & Asociación de Psicólogos de Buenos Aires (APBA) \\
\hline June 1963 & 1986 & Asociación de Psicólogos de La Plata *** \\
\hline 1971 & 1975 & Confederación de Psicólogos de la República Argentina (COPRA) \\
\hline 1977 & - & Federación de Psicólogos de la República Argentina (FePRA) \\
\hline \multicolumn{3}{|r|}{ Instituciones Académicas } \\
\hline 1991 & - & Asociación de Unidades Académicas de Psicología de Argentina y Uruguay (AUAPsi) **** \\
\hline 2003 & & $\begin{array}{l}\text { Unidad de Vinculación Académica de Psicología de Universidades de Gestión Privada } \\
\text { (UVAPsi) ***** }\end{array}$ \\
\hline \multicolumn{3}{|c|}{ * Aun cuando existen escasos registros de su actividad con posterioridad a 1973, ha existido hasta la actualidad. } \\
\hline \multicolumn{3}{|c|}{ ** Desde 1985 pasó a ser el Colegio de Psicólogos de Santa Fé, con dos circunscripciones. } \\
\hline \multicolumn{3}{|c|}{ *** Desde 1986 se subsumió en el Colegio de Psicólogos de la Provincia de Buenos Aires, Distrito XI. } \\
\hline \multicolumn{3}{|c|}{ **** Nuclea a las carreas de psicología de universidades de gestión pública. } \\
\hline \multicolumn{3}{|c|}{$\begin{array}{l}\text { ***** Nuclea a las carreras de psicología de universidades de gestión privada. Inicialmente se denominó Asociación de Unida- } \\
\text { des Académicas de Psicología de Universidades Privadas (AUAPri). }\end{array}$} \\
\hline
\end{tabular}


diversidad teórica y ha intentado cubrir con mayor amplitud nuevas áreas de la práctica profesional, todavía está fuertemente sesgada por las instituciones y la perspectiva psicoanalíticas.

También las organizaciones de la psicología han evidenciado ese sesgo, aun cuando desde la recuperación democrática se ha verificado el surgimiento de nuevas sociedades e instituciones críticas de la tradición únicamente profesionalista y clinicista (Tabla 12).

Especialmente la Asociación Argentina de Ciencias del Comportamiento (AACC) y la Asociación para el Avance de la Ciencia Psicológica (AACP) constituyen organizaciones fuertemente críticas de la tradición heredada en la psicología profesional argentina. La AACC, importante porque es la representante nacional en IUPsyS, representa especialmente a los investigadores y becarios del CONICET. La AACP, por su parte, nuclea también a investigadores del CONICET y sobre todo jóvenes becarios, doctorandos y maestrandos de las nuevas generaciones. Se caracteriza por ser una institución con delegaciones en distintas zonas del país y por ser expresión de los sectores más jóvenes, dinámicos y cuestionadores de la psicología profesionalista tradicional. En esa dirección, esas organizaciones se han preocupado tanto por los avances empíricos y contrastables en la investigación como por el énfasis en la responsabilidad pública y social de la psicología y los psicólogos y psicólogas.

Sintetizando, la psicología argentina se encuentra en una encrucijada con solamente dos caminos. Por uno de ellos, se vuelve a transitar la estrechez que caracterizó a una psicología encerrada sobre sí misma y autorizada únicamente en la lectura de los clásicos, como corresponde a las humanidades. Por el otro, sin desdeñar la enseñanza que autores clásicos pueden seguir aportando, se procura construir una ancha avenida propia de las ciencias sociales y naturales, con avances, retrocesos e inclusive contradicciones, pero siempre orientados por la necesidad de combinar la investigación empírica rigurosa en la psicología con la relevancia social de la misma en la esfera pública. En ese camino, uno de los principales desafíos es reexaminar el perfil de las carreras de psicología de manera tal que sus egresados puedan contribuir al avance de la ciencia y al mejoramiento de la calidad de vida de los ciudadanos y las ciudadanas.

\section{Referencias}

Alonso, M., \& Klinar, D. (noviembre, 2014). Los psicólogos en Argentina. Relevamiento cuantitativo 2013. Trabajo presentado en el VI Congreso Internacional de Investigación y Práctica Profesional en Psicología, XXI Jornada de Investigación y $10^{\circ}$ Encuentro de Investigadores de Psicología del MERCOSUR. Manuscrito inédito, Universidad de Buenos Aires, Facultad de Psicología.

Anónimo. (1954). Primer Congreso Argentino de Psicología. Humanidades, 4, 121-122.

Benito, E. (2009). La formación en psicología. Psiencia. Revista Latinoamericana de Ciencia Psicológica, 1(2), 3-10.

Bongiovani, P. C., \& Nakano, S. (2011). Acceso abierto en argentina: la experiencia de articulación y coordinación institucional de los repositorios digitales en ciencia y tecnología. e-colabora. Revista de ciencia, educación, innovación y cultura apoyadas por redes de tecnología avanzada, 1(2), 163-179.

Biagini, H. (1992). Historia ideológica y poder social. Buenos Aires: Centro Editor de América Latina.

Bleger, J. (1962). Clase inaugural de la cátedra de Psicoanálisis. Acta Psiquiátrica y Psicológica Argentina, 8(1), 56-60.

Bricht, S., Calvo, I., Dimant, F., Pravaz, S., Calvo de Spolanky, M., Troya, E. ... Palacios, I. (1973). El rol del psicólogo. Buenos Aires: Nueva Visión.

Calabresi, C., \& Polanco, F. (2011). Education in psychology in Argentina. Hugo Klappenbach's interview. Universitas Psychologica, 10(2), 613-626.

Coll, C. (1994). Posibilidades críticas en el desarrollo de la reforma curricular española. Substratum, 2(5), 125-150.

Comisión Nacional de Evaluación y Acreditación Universitaria. (1998). Creación de subsedes universitarias. Declaración de la Comisión Nacional de Evaluación y Acreditación Universitaria. Recuperado de http://www.coneau.edu.ar/archivos/1217.pdf 
Comisión Nacional de Evaluación y Acreditación Universitaria. (2014). Posgrados acreditados de la República Argentina. Edición 2014. Buenos Aires: Autor.

Constitución de la Nación Argentina. [1949]. En Cámara de Diputados de la Nación (Ed.), Digesto constitucional de la Nación Argentina (pp. 7-42). Buenos Aires: Imprenta del Congreso de la Nación.

Courel, R., \& Talak, A. M. (2001). La formación académica y profesional del psicólogo en Argentina. En J. P. Toro \& J. F. Villegas (Eds.), Problemas centrales para la formación académica y el entrenamiento profesional del psicólogo en las Américas (Vol. 1, pp. 21-83). Buenos Aires: Sociedad Interamericana de Psicología.

Dagfal, A. (2009). Entre París y Buenos Aires. La invención del psicólogo (1942-1966). Buenos Aires: Paidós.

De la Cruz, S. M. (2010). Hacia la acreditación de la carrera de Psicología. En E. H. Becerra \& D. L. Oliva (Eds.), Consideraciones sobre acreditación, mercantilización y regulación en las universidades latinoamericanas (pp. 65-83). San Luis: Ediciones LAE -Laboratorio de Alternativas Educativas.

Di Doménico, C. (en prensa). Formación de psicólogos: reflexiones sobre los procesos de acreditación en Argentina. En P. Altamirano (Ed.), Formación en Psicología en Argentina. Desafíos para la formación del psicólogo en el S.XXI. Córdoba: Universidad Nacional de Córdoba.

Di Doménico, C., \& Piacente, T. (2003). Acreditación de carreras de psicología en Argentina. Estado actual y perspectivas. En P. Marassi, J. P. Toro \& J. F. Villegas (Eds.), Problemas centrales para la formación de los psicólogos en las Américas (Vol. 3, pp. 31-57). Santiago de Chile: Sociedad Interamericana de Psicología.

Di Doménico, C., \& Piacente, T. (2011). Acreditación en Psicología en el Cono Sur de América. Psicolatina, 22, 1-18.

Di Doménico, C., \& Risueño, A. (2013). Procesos de acreditación de carreras de psicología en Argentina. Estado actual y prospectiva. Integración Académica en Psicología. Revista científica y profesional de la Asociación Latinoamericana para la Formación y la Enseñanza de la Psicología, 1(2), 24-28.
Edelmuth, D. (1997). Los consejeros en orientación. En L. Rossi (Ed.), La psicología antes de la profesión (pp. 157-166). Buenos Aires: Eudeba.

Fanelli, A. M. G., de \& Balán, J. (1994). Expansión de la oferta universitaria: nuevas instituciones, nuevos programas [Documento CEDES/106]. Buenos Aires: Centro de Estudios de Estado y Sociedad.

Fernández-Álvarez, H. (2003). [Presentación]. En J. F. Villegas, P. Marassi \& J. P. Toro (Eds.), Problemas centrales para la formación de los psicólogos en las Américas. (Vol. 3, pp. 13-16). Santiago de Chile: Sociedad Interamericana de Psicología.

Ferrari, F. (2013). Virgilio Ducceschi y el primer laboratorio de psicología experimental en Córdoba, Argentina (1907). Estudos e Pesquisas em Psicologia, 13(2), 779-805.

Ferrero, A., \& de Andrea, N. (2011). Regulación de la psicología por parte del Estado en la República Argentina. Revista Electrónica de Psicología Política, 9(26), 68-73.

Instituto Nacional de Estadísticas y Censos. (2005). Censo Nacional Económico. Operativo especial para profesionales. Recuperado de www.indec.mecon.ar/ economico2005/cne_08_05.pdf

Instituto Nacional de Estadísticas y Censos. (2012). Censo nacional de población, hogares y viviendas 2010. Censo del bicentenario. Resultados definitivos (Vol. 1). Buenos Aires: Autor.

Ingenieros, J. (1910). La psicología en la República Argentina. Anales de Psicología, 1, 343-350.

Klappenbach, H. (2000). El título profesional de psicólogo en Argentina. Antecedentes históricos y situación actual. Revista Latinoamericana de Psicología, 32(3), 419-446.

Klappenbach, H. (2003). La globalización y la enseñanza de la psicología en Argentina. Psicologia em Estudo, 8(2), 3-18.

Klappenbach, H. (2006). Periodización de la psicología en Argentina. Revista de Historia de la Psicología, 27(1), 109-164.

Klappenbach, H., \& Arrigoni, F. (2011). Revista Argentina de Psicología 1969-2002. Estudio bibliométrico. Revista Argentina de Psicología, 50, 44-94.

Lewis, P. H. (1990). The crisis of Argentine capitalism. Chapel Hill, NC: The University of North Carolina Press. 
Ley 14.184 de 1952. Segundo Plan Quinquenal de la República Argentina. Diciembre 29 de 1952. BO. N. 17327.

Ley 24.521 de 1995. Ley de Educación Superior de la República Argentina. Julio 20 de 1995. BO N.음 28.204.

Litvinoff, N., \& Gomel, S. K. de (1973). El psicólogo y su profesión. Buenos Aires: Nueva Visión.

Marano, G. (2010). iHacia una universidad pulpo? La apertura de sedes: expansión, tramas políticas y mercado universitario. Revista Argentina de Educación Superior, 2(2), 10-36.

Mercado, B. E. (2006). La psicología académica en Argentina. Trayectoria de la gestión privada y protagonismo de la Universidad del Salvador. Signos, 25, 105-117.

Ministerio de Educación, Ciencia y Tecnología. (2007). Anuario 2006 de Estadísticas Universitarias. Buenos Aires: Autor.

Ministerio de Educación. (2014). Base de títulos oficiales. Buenos Aires: Autor. Recuperado de http://titulosoficiales.siu.edu.ar/

Moya, L. (2010). Estudio comparado de la formación básica en psicología en carreras de universidades públicas nacionales. Anuario de Proyectos e Informes de Becarios de Investigación, 7, 357-361.

Noé, A. (2005). Utopía y desencanto. Creación e institucionalización de la Carrera de Sociología en la Universidad de Buenos Aires: 1955-1966. Buenos Aires: Miño y Dávila.

Pérez-Lindo, A. (2007). Prospectiva de la educación superior argentina 2020. Buenos Aires: Secretaría de Ciencia y Tecnología.

Pérez-Rasetti, C. (2008). Morfología de la expansión universitaria: estrategias institucionales de expansión geográfica en las universidades argentinas. Gestión Universitaria, 1 (1). Recuperado de http:// www.gestuniv.com.ar/gu_01/v1n1a1.htm

Pérez-Rasetti, C., \& Araujo, J. (diciembre, 2010). Expansión de la educación superior en la Argentina: desafíos y propuestas para pensar las políticas de acceso. Trabajo presentado en el X Coloquio Internacional sobre Gestión Universitaria en América del Sur, Universidad Nacional de Mar de Plata, Argentina. Recuperado de https://repositorio.ufsc. br/handle/123456789/96624
Piñeda, M. A. (2006). Antecedentes políticos y académicos de la creación de la carrera de psicología en las primeras universidades católicas argentinas. Psicología y Psicopedagogía, 5(14). Recuperado de http://p3.usal.edu.ar/index.php/psico/article/ view/1278/1633; 14/06/2007

Resolución CONEAU 1028 de 2013 [Comisión Nacional de Evaluación y Acreditación Universitaria Expte. № 804-1600/11]. Acreditar con compromisos de mejoramiento la carrera de Licenciatura en Psicología de la Facultad de Psicología y Relaciones Humanas de la Universidad Abierta Interamericana Sede Ciudad Autónoma de Buenos Aires por un periodo de tres años. 9 de diciembre de 2013. Recuperado de http://www.coneau.gov.ar/ archivos/resoluciones/Res1028-13E804160011.pdf

Resolución CONEAU 1018 de 2013 [Comisión Nacional de Evaluación y Acreditación Universitaria Expte. № 804-1621/11]. Acreditar la carrera de Licenciatura en Psicología de la Facultad de Psicología y Ciencias Sociales de la Universidad de Ciencias Empresariales y Sociales Sede Ciudad Autónoma de Buenos Aires por un periodo de seis años. 9 de diciembre de 2013. Recuperado de http://www.coneau.gov.ar/archivos/resoluciones/ Res1018-13E804162111.pdf

Resolución CONEAU 1041 de 2013 [Comisión Nacional de Evaluación y Acreditación Universitaria Expte. № 804-1771/11]. Acreditar con compromisos de mejoramiento la carrera de Licenciatura en Psicología de la Facultad de Psicología y Ciencias Sociales de la Universidad de Ciencias Empresariales y Sociales Sede Rafaela por un periodo de tres años. 9 de diciembre de 2013. Recuperado de http://www.coneau.gov.ar/archivos/resoluciones/ Res1041-13E804177111.pdf

Resolución № 2447 de 1985 [Ministerio de Educación y Justicia de la Nación]. Por la cual se eleva una propuesta de incumbencias profesionales correspondientes a los títulos de Psicólogo y Licenciado en Psicología. 20 de septiembre de 1985.

Resolución 136 de 2004 [Ministerio de Educación, Ciencia y Tecnología]. Declárase incluido en el régimen del artículo 43 de la Ley № 24.521, al título de Licenciado en Psicología. 23 de febrero de 2004. 
Resolución 343 de 2009 [Ministerio de Educación]. Apruébanse los contenidos curriculares básicos, la carga horaria, los criterios de intensidad de la formación práctica y los estándares para la acreditación de las carreras correspondientes a los títulos de Psicólogo y Licenciado en Psicología. 30 de septiembre de 2009.

Resolución 800 de 2011 [Ministerio de Educación]. Modifícase la Resolución N³43/09, relacionada con los contenidos curriculares básicos, la carga horaria, los criterios de intensidad de la formación práctica y los estándares para la acreditación de las carreras correspondientes a los títulos de Psicólogo y Licenciado en Psicología. 4 de mayo de 2011.

Resolución 160 de 2011 [Ministerio de Educación]. Apruébanse los estándares y criterios a considerar en los procesos de acreditación de carreras de posgrado. 29 de diciembre de 2011.

Scimago Research Group. (2011). Ranking iberoamericano de psicología SIR 2011. Recuperado de http:// www.scimagoir.com
Suasnábar, C., \& Rovelli, L. (2011). Políticas universitarias en Argentina: entre los legados modernizadores y la búsqueda de una nueva agenda. Revista Innovación Educativa, 11(57), 21-30.

Vezzetti, H. (2004). Los comienzos de la psicología como disciplina universitaria y profesional. En F. Neiburg \& M. Plotkin (Eds.), Intelectuales y expertos. La constitución del conocimiento social en la Argentina (pp. 293-326). Buenos Aires: Paidós.

Vilanova, A. (1993). La formación de psicólogos en Iberoamérica. Acta Psiquiátrica y Psicológica de América Latina, 39(3), 193-205.

Visca, J. E. (2010). Acceso y uso de la información científica en estudiantes avanzados de carreras de la facultad de psicología y de la facultad de ciencias exactas de la UNMP. Estudio comparativo. Anuario de Proyectos e Informes de Becarios de Investigación, 7(2), 363-367.

Zelaya, M. (2012). La expansión de universidades privadas en el caso argentino. Pro-Posições, 23(2), 179-194. 
